\title{
La política exterior dependiente: el caso de Ecuador
}

\section{Ramiro Lapeña Sanz ${ }^{*}$ Marcin Roman Czubala Ostapiuk*}

\section{RESUMEN}

La política exterior dependiente es un concepto que define la relación de un Estado con otras naciones. Dicha correlación, traducida en una estrecha vinculación de su economía frente a uno o varios países, puede ser ocasionada de manera voluntaria o contra el deseo del Estado en cuestión, incidiendo así en su política exterior. Partiendo de este supuesto, el objetivo principal del presente trabajo es analizar el caso particular de Ecuador mediante un examen en profundidad de su relación con Estados Unidos de América (eE.uu.) y República Popular China. Igualmente, el marco temporal de este artículo está centrado en el período 20072016, cubriendo así el mandato del presidente Rafael Correa.

Para ello, en primer lugar, se expone el marco teórico y el estado de la cuestión. Posteriormente, se estudian los intercambios económicos de Ecuador con Estados Unidos de América y República Popular China, poniendo un especial énfasis en las relaciones comerciales, la inversión extranjera directa, la deuda y la ayuda oficial al desarrollo. Por último, se presentan las conclusiones obtenidas.

Palabras clave: Ecuador, dependencia económica, política exterior dependiente, Estados Unidos, China

* Doctor (c) en periodismo y MsC en política internacional: estudios sectoriales y de área, ambas por la Universidad Complutense de Madrid (UCM). Docente de geopolítica en la Facultad de Ciencias Administrativas de la Universidad uTE sede Santo Domingo, (Ecuador) [ramiro.lapena@ute.edu.ec].

** Doctor en ciencias políticas por la Universidad Complutense de Madrid, Premio Extraordinario de Doctorado (España). Director académico y profesor de cedeu Centro de Estudios Universitarios, adscrito a la Universidad Rey Juan Carlos de Madrid, (España) [m.romanczubala@cedeu.es].

Recibido: 21 de abril de 2017 / Modificado: 27 de febrero de 2018 / Aceptado: 7 de junio de 2018

Para citar este artículo:

Lapeña Sanz, R. y Czubala Ostapiuk, M. R. (2018). La política exterior dependiente: el caso de Ecuador. OASIS, 28, pp. 171-191.

DoI: https://doi.org/10.18601/16577558.n28.10 


\section{The dependent foreign policy: the case of Ecuador}

\section{ABSTRACT}

The dependent foreign policy is a concept that defines the relationship of a State with other partners. Translated into a close linkage of its economy with those countries, it may be caused voluntarily or against the will of the State in question, thus influencing its foreign policy. Starting from this assumption, the main objective of this article is to analyze the case of Ecuador, examining in depth its relation with the United States of America and the People's Republic of China. Likewise, the time frame is centered in the period between 2007 and 2016, thereby covering the mandate of President Rafael Correa.

To accomplish the mentioned goal, first we review the theoretical framework and the state in question. Secondly, we analyze Ecuador's economic exchanges with the USA and China, with a special emphasis on trade relations, foreign direct investment, debt and official development assistance. Finally, the obtained conclusions will be presented.

Key words: Ecuador, Economic dependence, Dependent Foreign Policy, United States, China

\section{INTRODUCCIÓN}

De acuerdo con la teoría de política exterior dependiente (Dependent Foreign Policy Theory) la actividad de un país en la materia se define como "el comportamiento internacional de
Estados económicamente débiles cuyas economías dependen en gran medida de uno o dos socios comerciales" (Hey, 1994, p. 241). Traducida en una estrecha vinculación entre dos actores, dicha subordinación puede ser ocasionada de manera voluntaria o contra el deseo del Estado en cuestión, incidiendo así en su política exterior.

Igualmente, y con arreglo a la teoría desarrollada, se establece una correlación entre dos tipos de países. En primer lugar, un Estado pequeño (small state) o débil (weak state). En segundo lugar, un país grande o desarrollado (core state). Además, tampoco debemos olvidar que para poder hablar acerca de una política exterior dependiente es necesaria una subordinación económica real. De esta manera, el nexo entre ambos actores se establece tanto en el ámbito de comercio, las inversiones extranjeras directas y los créditos como de las ayudas económicas, entre otros.

Mediante su trabajo Jeanne A. K. Hey (1995) realizó un análisis de las presidencias de Osvaldo Hurtado (1981-1984) y León Febres Cordero (1984-1988) en Ecuador. En esta investigación no solo tomó a este país como un caso de estudio sobre el fenómeno de política exterior dependiente, sino también detectó una serie de comportamientos típicos para dicho Estado, haciendo hincapié en los estrechos vínculos del país andino respecto a Estados Unidos de América.

Así, la economía ecuatoriana, basada principalmente en la exportación de productos agrícolas y ganaderos no procesados, junto con la escasez de su sector industrial y la baja competitividad, mostró un alto grado de debilidad en la etapa como república independiente frente 
al resto del mundo. En otras palabras, un reflejo de una serie de características muy comunes para el conjunto de los países de la región. Además, Estados Unidos y los países europeos, los principales socios comerciales de Ecuador, tampoco contribuyeron al aumento de las posibilidades del desarrollo de su sector productivo, ya que exportaban al país andino los bienes de equipo, intermedios y de consumo final necesarios.

Asimismo, conviene recordar que el Estado norteamericano, en su momento, llevó a cabo una serie de acciones enfocadas no solo en la contención del comunismo, sino que también entregó ayuda financiera destinada para el conjunto de la región y de la que Ecuador ha sido beneficiario. Además, la interferencia estadounidense en su conjunto ha sido muy visible, afectando a la soberanía del país andino en el marco de su política exterior hasta el 2007 (Lauderbaugh, 2009; Pineo, 2009).

Sin embargo, el cambio de gobierno y la llegada al poder de Rafael Correa dieron lu- gar a una alteración de perspectiva, buscando convertir al país en cuestión en un Estado con un lugar propio en el mundo, rompiendo de esta manera con el statu quo preestablecido y protegiendo la soberanía nacional (Ministerio de Relaciones Exteriores, Comercio e Integración de la República de Ecuador, 2007). Como señalase en su discurso de aceptación del cargo de ministro de relaciones exteriores Ricardo Patiño (2010-2016), la revolución ciudadana desde sus inicios buscó transformar la política exterior del país desde dos principios: "la soberanía nacional y la dignidad del pueblo ecuatoriano" (Patiño, 2010).

De esta manera la nueva política exterior del gobierno de Rafael Correa buscó frenar el estrechamiento de las relaciones bilaterales con su socio norteamericano, acercándose a las demás naciones del Cono Sur (Venezuela y Nicaragua) y de otros continentes (p.ej. Irán, China ${ }^{1}$ o Rusia, entre otros) con la premisa de fomentar el desarrollo interno del país ${ }^{2}$.

1 Rafael Correa visitó China en enero de 2015, convirtiéndose en el primer presidente de Ecuador en hacerlo en toda la historia del país, demostrando así la importancia que el gobierno Correa daba a Beijing como socio político y económico.

2 Las principales modificaciones introducidas a través de un plan de desarrollo económico contaron con la diversificación de la economía, el fomento de la producción de bienes de consumo finales, así como el establecimiento de una industria más competitiva, basada en educación y la salud, dando así el giro a la matriz productiva de Ecuador (Secretaría Nacional de Planificación y Desarrollo, 2013). Con ello se pretendía diversificar y ampliar productos y mercados, pues la concentración en unos pocos artículos y/o en unas pocas plazas comerciales hacía al país frágil y dependiente (Patiño, 2010). Dentro de este plan se incluía una nueva visión respecto a la política exterior del Estado. Fundada en el Planex 2020-Plan de Política Exterior 2006-2020 (Ministerio de Relaciones Exteriores de la República de Ecuador, 2006) y el Plan Nacional de Desarrollo 2007-2010 (Ministerio de Relaciones Exteriores, Comercio e Integración de la República de Ecuador, 2007), así como plasmada en la Constitución de Montecristi de 2008, la política exterior de Ecuador renovada tenía como objetivo reducir su dependencia de los Estados Unidos de América y aportar en cambio hacia la conformación de un orden multipolar a través de participar en bloques económicos o políticos regionales (Patiño, 2010), tales como la Unión de Naciones Suramericana (Unasur), la Alianza Bolivariana para los Pueblos de Americana Latina (Alba), e incluso iniciativas como el Banco del Sur. 
Partiendo de este supuesto, el presente trabajo persigue analizar el caso de Ecuador, examinando la relación entre la posible presencia de dependencia económica y su influencia sobre la acción exterior del Estado. Asimismo, su desarrollo permite verificar la hipótesis: bajo el mandato de Rafael Correa, Ecuador presentó dependencia económica no solo frente a Estados Unidos de América, sino también con República Popular China.

Teniendo en cuenta la enorme heterogeneidad del estudio realizado y buscando comprender mejor la realidad analizada, se decidió basar su desarrollo en el análisis detallado de la literatura del tema (tanto nacional como internacional), así como otras fuentes de información, entre ellas declaraciones públicas en distintos soportes físicos y electrónicos realizadas por miembros del gobierno Correa, fomentando así la calidad de la investigación procedida. Igualmente, el presente estudio, de tipo correlacional, cuenta con la revisión documental de datos de comercio, ayuda económica, créditos y préstamos e inversión extranjera directa extraídos de diferentes fuentes primarias: Banco Central del Ecuador (BCE), Comisión Económica para América Latina y Caribe (Cepal), Conferencia de las Naciones Unidas sobre Comercio y Desarrollo (Unctad), Organización Mundial de Comercio (oMc), Banco Mundial (BM) y Fondo Monetario Mundial (FMI).

El marco temporal, centrado en el período 2007-2016, cubre el mandato del presidente Rafael Correa Delgado. No obstante, y con el objetivo de proceder a una evaluación más completa, se ampliaron algunas partes del tra- bajo presentado, buscando ofrecer una mayor capacidad explicativa longitudinal.

Para ello, en primer lugar, se expone el marco teórico y el estado de la cuestión. Posteriormente, se estudian los intercambios económicos de Ecuador con Estados Unidos de América y República Popular China, poniendo un especial énfasis en las relaciones comerciales, la inversión extranjera directa, la deuda y la ayuda oficial al desarrollo. Por último, se presentan las conclusiones obtenidas.

\section{MARCO TEÓRICO}

Desde el inicio de la guerra fría las relaciones internacionales han visto una singular profusión de estudios sobre análisis de política exterior. No obstante, en su gran mayoría el objeto primordial de dichas investigaciones han sido las potencias del sistema internacional de la época (los Ee.uU., el Reino Unido, la Unión de Repúblicas Socialistas Soviéticas, Francia, etc.), siendo muy pocos los estudios que abordan como objeto a Estados de menor tamaño y/o importancia dentro del sistema existente. En este sentido, la política exterior de dichos Estados (más pequeños o más débiles) tradicionalmente en las RR.II. ha sido tratada desde el paradigma realista, colocando a estos actores en una posición de subyugación frente al poder militar, económico y político de las grandes potencias.

Más adelante, y a través de la teoría de la dependencia (Dependency theory) y la teoría del sistema-mundo (World-System Theory), el estructuralismo aportaría nuevas herramientas, una visión diferente sobre la distribución de la 
organización de Estados, poniendo a la economía como centro del análisis. A partir de ahí el sistema internacional quedó caracterizado al colocar en el núcleo (core) de la ordenanza a los países con mayor desarrollo económico y a los Estados menos desarrollados o pobres en su periferia (Steans, 2010, pp. 75-102).

De acuerdo con lo establecido, la desigualdad y el desequilibrio de las relaciones centro-periferia están causadas en tanto que el contorno actúa como proveedor de materias primas, mano de obra barata y comodidades requeridas por los países del centro. Una situación causada por su alto nivel de industria, tecnología, innovación y desarrollo. Asimismo, la riqueza del núcleo se logra a través de la pobreza de la periferia, pues el sistema está diseñado para favorecer los intereses de los Estados más ricos (Steans, 2010, p. 83).

Sin embargo, la perspectiva de las teorías estructuralistas no era capaz de explicar las políticas exteriores de los países de la periferia. De ahí que desde los años 60 un reducido grupo de académicos (Neil Richardson, Bruce Moon, Jeanne A. K. Hey y otros) se centrase en articular una teoría de política exterior dependiente (Dependent Foreign Policy Theory) que pudiera servir para el análisis de estas naciones del contorno, cuyas tipologías siguen sin definir. Este último punto es conflictivo, pues a menudo los términos de Estado pequeño (small state), Estado débil (weak state), Estado del Tercer Mundo (Third World state) y Estado dependiente (dependent state) se emplean como sinónimos cuando existen diferencias conceptuales entre unos y otros.

Un Estado pequeño puede serlo en territorio, pero quizá no en aspectos demográficos, económicos o de fuerza militar, p.ej. las repúblicas bálticas o Islandia. Un Estado débil puede presentar dicha característica ante una falta de gobernabilidad o una economía extremadamente dependiente de ayuda exterior, pese a ser muy grande en extensión (el caso del Estado de Libia actual), mientras que la categoría de tercermundista se le da merced a una variable geopolítica, el lugar que ocupa en el globo y el poder que tienen esos países económicamente hablando, que suele coincidir con posiciones de periferia.

Para Hey (1995a) cada uno de estos conceptos no logra relacionar la situación de los Estados con la formulación y puesta en marcha de su política exterior, a excepción del término Estado dependiente.

En este punto se debe aclarar la disimilitud entre dos vocablos que en castellano son uno solo, dependencia, pero que en inglés tienen dos sentidos distintos, dependence y dependency, este último usado específicamente bajo el paradigma estructuralista. La diferencia entre ambos reside en el enfoque y los métodos de estudio. El planteamiento de la dependence es el de recoger el estado de confianza que un Estado tiene sobre otro, en tanto la dependency supone contemplar un conjunto más completo de relaciones que busca incorporar a la división internacional del trabajo a los países menos desarrollados y a las sociedades que presentan menor grado de homogeneidad (Hey, 1995a, p. 204).

Así, la política exterior dependiente (el principal eje teórico de este trabajo) puede definirse, siguiendo a Hey (1994, p. 241), como "el comportamiento internacional de Estados económicamente débiles cuyas economías dependen en gran medida de uno o dos socios comerciales". 
Esa conducta del país subordinado se traduce en una política exterior coincidente con los intereses del Estado del cual dependen, ya sea de forma voluntaria o en contra de su deseo. Con base en esa teoría se expone como uno de los requisitos de la existencia de una política exterior sometida que se dé una auténtica relación de dependencia económica entre el país pequeño (smallstate) o débil (weak state) y el Estado más grande o desarrollado (denominado en la literatura como core state). Dicha subordinación económica se manifiesta en campos como el comercio, las inversiones extranjeras directas, los créditos y las ayudas económicas o de otro tipo.

Por lo que respecta a la diferencia entre la dependence y dependency en los métodos de estudio, son las variables las que marcan la distinción. Las manejadas en la dependency son de carácter cualitativo (por ejemplo, la apertura de mercados) y por ello difíciles de cuantificar. En cuanto las variables de la dependence, estas son fácilmente medibles por ser variables económicas cuantitativas. Además, Hey destaca hasta tres indicadores identificativos de dependent states que precisan la dependencia económica de un país de la periferia con respecto a un Estado del centro: los flujos de comercio, la ayuda exterior y la inversión extranjera directa del país del centro (Hey, 1995ª, p. 204).

\section{ESTADO DE LA CUESTIÓN}

En efecto, los Estados periféricos suelen mostrar en todos los casos una dependencia crítica con una nación más desarrollada. En la región latinoamericana el país que ha ejercido ese papel último ha sido Estados Unidos de América, cuya política exterior hacia su hemisferio sur ha sido influida por la doctrina formulada en 1823 por el presidente James Monroe. Aunque la Doctrina Monroe estaba orientada a rechazar inicialmente la colonización europea de los territorios de América Latina en un momento de desmoronamiento del imperio español merced a los gritos de independencia, ha servido como justificativo para, entre otras cosas, proteger el orden y la estabilidad en áreas sensibles de los intereses de EE.UU. o asegurar el acceso sin obstáculos de sus productos a los mercados latinoamericanos (Sánchez Padilla, 2016, p. 14).

A partir de 1895 la Doctrina se invocó para justificar políticas cada vez más intervencionistas y agresivas en los países del hemisferio sur (Sánchez Padilla, 2016, p. 14). Especialmente tras la segunda guerra mundial y con la adopción, por el presidente Harry S. Truman, de la doctrina de la contención del comunismo planteada por George F. Kennan. Precisamente es en este período histórico en el que EE.UU. ha empleado la ayuda exterior, los créditos y la inversión extranjera como herramientas de poder blando para ejercer presión sobre los países periféricos del Centro y Suramérica, para que adoptasen políticas favorables a sus intereses.

La subordinación económica (el principal punto de análisis de esa investigación) es crítica al abordar la política exterior de los países periféricos, en especial en el caso de las naciones latinoamericanas. A la luz de la teoría de política exterior dependiente se observa cómo, mientras el terreno diplomático es el espacio en el que estos Estados pueden desenvolverse con mayor soltura y plantear comportamientos en política exterior que vayan en contra de los in- 
tereses de los países dominantes, el área económica es donde no existe ese mayor margen de maniobra para oponerse ya que normalmente los intereses de las naciones desarrolladas se encontrarían bajo amenaza. Esto lleva a la conclusión de que cualquier país que dependa del centro, sea este representado por Estados Unidos o por cualquier otra nación, solo podría ser capaz de diseñar e implementar una política exterior autónoma en las áreas que el Estado dominante considere poco importante (Hey, 1993, p. 573). En otras palabras, el margen de soberanía real que le queda a la nación dependiente es muy reducido, limitando con ello las opciones que tenga y condicionando las acciones de su política exterior.

Pese a que numerosos estudios de política exterior de América Latina han puesto a EE.UU. como el histórico país dominante sobre las naciones de esta región, en los últimos años se observa un incremento notable de la presencia de otro actor que emplea similares herramientas que las de Washington. El gigante asiático se ha insertado en la economía global de forma decidida desde el mandato de Den Xiaoping en los años ochenta. Hoy tiene buena parte de su sistema orientado al comercio exterior, al punto que el $22,1 \%$ del pIB de China en 2015 fue generado por exportaciones de bienes y servicios, aunque en años anteriores el porcentaje correspondiente había sido aún mayor -en 2006 la cifra fue de 37,17\% del PIB (Banco Mundial, 2017a); en forma global el comercio de bienes en el mismo año ascendió a 2,280 billones de dólares-.

La relación del gigante asiático con América Latina es igualmente intensa. El comercio de bienes en el 2015 desde la República Po- pular China hacia la región fue de 129.980,96 millones de dólares en exportaciones y de 103.625,11 en importaciones, representando una balanza comercial desfavorable (26.355 millones de dólares) para los países latinoamericanos con respecto al país asiático (Fondo Monetario Internacional, 2017). Los principales productos de importación del país asiático desde América Latina son minerales de hierro, soja y otras semillas oleaginosas, cobre y petróleo, sumando todo ello el 69\% de las exportaciones, mientras que en sentido contrario China envía a la región equipos para telecomunicaciones, máquinas de procesamiento de datos, barcos, instrumentos y aparatos de óptica y derivados del petróleo, que suman un $22 \%$ del total de importaciones (Ray, Gallagher \& Sarmiento, 2016, p. 3).

Por lo que respecta a la concesión de créditos por parte de Beijing a la región latinoamericana, en 2015 se transfirieron 29.000 millones de dólares. Un montante superior al que destinaron en conjunto el Banco Mundial, el Banco de Desarrollo de América Latina (CAF) y el Banco Interamericano de Desarrollo (BID), mientras que la inversión extranjera directa de la República Popular China en el área ascendió ese mismo año a 4.600 millones de dólares en proyectos nuevos y a 49,9 millones de dólares en fusiones y adquisiciones (Ray et al., 2016, pp. 2-4). De hecho, y de acuerdo con un reciente reporte del "Research from the Global Economic Governance Initiative", de la Universidad de Boston, el país asiático es ahora la segunda fuente más grande de proyectos IED nuevos detrás de los Estados Unidos de América, y el tercer impulsor principal de IED a través de fusiones y adquisiciones por detrás de los 
EE.UU. y España (Ray et al., 2016, p. 6). Los sectores en los que China invierte y concede créditos son fundamentalmente: transporte, energía hidráulica, petróleo y gas, minería, comunicaciones y logística; los dominios que están precisamente dentro del área de intereses de la economía de Beijing: energía, seguridad alimentaria, materia prima para su industria pesada y tecnológica y vías de comunicación logística para el comercio hacia y desde China a distintos países.

La irrupción de este Estado en el escenario regional propiciado, por otra parte, por una retirada parcial de EE.uU. al estar más centrado en escenarios de Oriente Medio y Asia-Pacífico, hace necesario plantearse si en el caso de Ecuador debemos hablar de una dependencia económica única tradicional (EE. uU.), o de una de tipo bidependiente, con el Estado norteamericano y China, con los países de los que se necesita en el ámbito económico.

\section{INTERCAMBIOS ECONÓMICOS CON ESTADOS UNIDOS Y CHINA}

A continuación se presentarán los datos acerca de las relaciones comerciales, de inversión, la deuda y de ayuda oficial al desarrollo que Ecuador tiene con República Popular China y Estados Unidos de América, y se llevará a cabo un análisis de los mismos desde el enfoque de la dependencia económica. La información se obtuvo mediante una revisión de fuentes primarias abiertas y disponibles, de tipo estadístico, localizadas en bases de datos de instituciones públicas y privadas de Ecuador (fundamentalmente el Banco Central del Ecuador (вСЕ), y el Ministerio de Finanzas y el Observatorio de Política Fiscal), así como en bases de datos de organismos internacionales como el Fondo Monetario Internacional, el Banco Mundial, la Comisión Económica para América Latina y Caribe (Cepal) y/o el sistema WITS $^{3}$.

\subsection{Comercio}

El volumen de exportaciones que Ecuador realizó al mundo durante 2016 fue de 16.797 millones de dólares en valor $\mathrm{FOB}^{4}$, mientras que las importaciones realizadas en dicho período por el país ascendieron a 15.545,2 millones de dólares, lo que deja una balanza comercial superavitaria de 1.252.5 millones de dólares (Banco Central del Ecuador, 2017b).

Respecto a las zonas geográficas, América es el principal socio comercial de Ecuador con el $60 \%$ del total de exportaciones realizadas por el país andino y el $51,1 \%$ de las importaciones efectuadas por este, lo siguen a bastante

3 El programa informático "Solución Comercial Integrada Mundial” (wITs, por sus siglas en inglés) fue creado por el Banco Mundial en colaboración con la Conferencia de las Naciones Unidas sobre Comercio y Desarrollo (UNCTAD) para poner a disposición de los usuarios información sobre comercio y aranceles, obtenida a partir de bases de datos de instituciones internacionales gubernamentales y entidades privadas.

4 FОв corresponde a las siglas del Incoterm 2010 conocido como Free on Board o Franco a Bordo. En estas condiciones el valor no contempla ni el flete del medio de transporte que va a llevar la mercancía a su destino final, ni tampoco el coste del seguro de la misma (International Chamber of Commerce, 2010). 
distancia Europa $(22,2 \%$ de las exportaciones y $13,3 \%$ de las importaciones) y Asia (16,9\% de exportaciones y $26 \%$ de las importaciones) (Banco Central del Ecuador, 2017b).

Si analizamos dicha relación en función de Estados, EE.uU. y China resultan presentar los mayores intercambios con Quito. De hecho, dominan las relaciones comerciales de Ecuador con sus respectivas áreas geográficas, América y Asia. En el caso de Estados Unidos, el socio norteño aglutina el 53,6\% de las exportaciones y el $42,3 \%$ de las importaciones totales de América hacia Ecuador, mientras que la República Popular China, por su parte, representa el 59,7\% de las importaciones y el 23,1\% de las exportaciones totales del país andino con el continente asiático (Banco Central del Ecuador, 2017b).

En 2016 Ecuador exportó a EE.uU. el $32,4 \%$ (5.436,1 millones de dólares) del volumen total e importó el $25,1 \%$ del total (3.896,9 millones de dólares). Las exportaciones petroleras representaron el 51,8\%, mientras que las no petroleras fueron del $23 \%$, en tanto que las importaciones se repartieron entre un $66,5 \%$ y un $17,2 \%$ respectivamente (Banco Central del Ecuador, 2017b). De hecho, y de acuerdo con los datos del BCE, EE.UU. fue el socio con el que Ecuador tuvo el mayor superávit comercial del sector (1.172 millones de dólares) y el segundo superávit comercial no petrolero (366,3 millones). Entre las principales materias enviadas por el país andino a EE.UU. podemos destacar: productos primarios sin elaborar (mariscos, banano, flores, cacao) y algunos rubros de industrias agroalimentarias, así como conservas de pescado. Asimismo, recibe de aquel derivados del petróleo como gasolinas, gas de petróleo y aceites y lubricantes, además de maquinaria industrial, teléfonos, instrumental médico-quirúrgico y polímeros (Banco Central del Ecuador, 2017b).

En lo concerniente a la relación de Ecuador con República Popular China, el mismo año se mandó al país asiático un 3,9\% de las exportaciones totales ecuatorianas $(656,4 \mathrm{mi}-$ llones de dólares), mientras que las importaciones ascendieron a un 15,5\% del total $(2.415$ millones de dólares). Dentro del primer grupo existe muy poca diferencia entre los bienes petroleros y no petroleros, puesto que los primeros representaron un 4,25\% (232 millones de dólares) y los segundos un 3,7\% (424,4 millones de dólares) del total. Sin embargo, sí existe una notable distancia entre las importaciones de hidrocarburos (1,4\%, 34,9 millones) y las no petroleras $(18,2 \%, 2.380,9$ millones de dólares) con respecto al conjunto de importaciones. De hecho, China es el cuarto país con el que tiene superávit comercial petrolero (197,1 millones de dólares), pero es al mismo tiempo la nación con la que Ecuador tiene el mayor déficit comercial fuera de este sector (1.965,5 millones de dólares). Descendiendo al análisis de la balanza comercial por productos, el país asiático recibe de Ecuador crudo, harinas, cacao, bananas, mariscos, madera y productos derivados de ella y productos de la industria minera (metales preciosos y cobre), en tanto que Ecuador importa de China motores y generadores eléctricos, teléfonos móviles, maquinaria, material eléctrico, monitores y proyectores, turbinas hidráulicas y neumáticos (Banco Central del Ecuador, 2017b). 
Tabla 1

Las relaciones comerciales de Ecuador con Estados Unidos de América y con República Popular China en el 2016

\begin{tabular}{|l|c|c|}
\hline & EE.UU. & China \\
\hline Exportaciones & $25,1 \%$ & $3,9 \%$ \\
\hline Exportaciones petroleras & $51,8 \%$ & $4,25 \%$ \\
\hline Exportaciones no petroleras & $23 \%$ & $3,7 \%$ \\
\hline Importaciones & $32,4 \%$ & $15,5 \%$ \\
\hline Importaciones petroleras & $66,5 \%$ & $1,4 \%$ \\
\hline Importaciones no petroleras & $17,2 \%$ & $18,2 \%$ \\
\hline
\end{tabular}

Fuente: Elaboración propia con base en el Banco Mundial. Recuperado a partir de http://datos.bancomundial.org/pais/ecuador?view=chart

A la luz de estos datos se concluye que a nivel comercial Estados Unidos de América continúa siendo la principal referencia para las empresas ecuatorianas dedicadas a la actividad exterior. Casi un tercio de todo lo exportado y un cuarto de lo importado por el país andino tienen su destino u origen en EE.UU., con un peso muy destacado del sector petrolero. Aun así, se observa cierto desequilibrio en los intercambios, pues pese a tener superávit comercial los productos ecuatorianos que viajan al mercado norteamericano son bienes primarios con poco o ningún valor añadido y crudo, un producto con una volatilidad de precios que tiene impacto directo en las cuentas nacionales, mientras que lo que entra por sus aduanas desde el país del norte son fundamentalmente productos refinados del petróleo ${ }^{5}$, bienes de equipo y equipamiento médico. En este sentido el gobierno de Rafael Correa ha intentado dar la vuelta a esta situación mediante políticas tendentes al cambio de la matriz productiva, haciéndola orientarse hacia productos de alto valor añadido, industriales y tecnológicos, buscando así reforzar su posición exportadora y dejar atrás su rol de mero importador de esos bienes.

No obstante, con República Popular China los flujos comerciales no resultan ser tan bidireccionales. El volumen de las importaciones es mucho más amplio, en especial las no petroleras,

5 Las refinerías más antiguas de Ecuador, como la de Salinas, en el suroeste del país, son incapaces de producir gasolinas de alto octanaje como la súper, y gran parte de este combustible debe entrar importado, y los subproductos obtenidos en ella son de mala calidad, de acuerdo con un responsable de la refinería La Libertad de Petroecuador en una visita que realizó uno de los autores con un grupo de alumnos a la planta, y corroborado por el propio Ministerio Coordinador de Sectores Estratégicos en la Agenda Nacional de la Energía 2016-2040 (ver Ministerio Coordinador de Sectores Estratégicos, 2016). 
y la balanza comercial es claramente deficitaria, merced al desequilibrio aún más acusado de los intercambios comerciales con respecto al caso norteamericano. Los artículos del sector primario enviados al gigante asiático tienen un valor inferior con respecto a bienes de equipo, componentes y productos tecnológicos que se importan desde ella. De esta forma, y en especial en su relación comercial, se refleja la penetración del gigante asiático en los mercados latinoamericanos debido, entre otros factores, a la mayor competitividad por precios de sus manufacturas.

\subsection{Inversión extranjera directa}

La inversión extranjera directa recibida por Ecuador ascendió en el 2015 a 1.321 millones de dólares, un 41,6\% más que en 2014, cuando sumó 771 millones de dólares. En referencia al 2016 los datos disponibles (hasta el tercer trimestre) muestran un monto de IED de 341 millones de dólares, lo que representa una caída del 58\% (197,9 millones de dólares) con respecto al mismo período del año anterior (Banco Central del Ecuador, 2017a) (Banco Central del Ecuador, 2017a).

Gráfico 1

La inversión extranjera directa recibida por Ecuador en el 2015

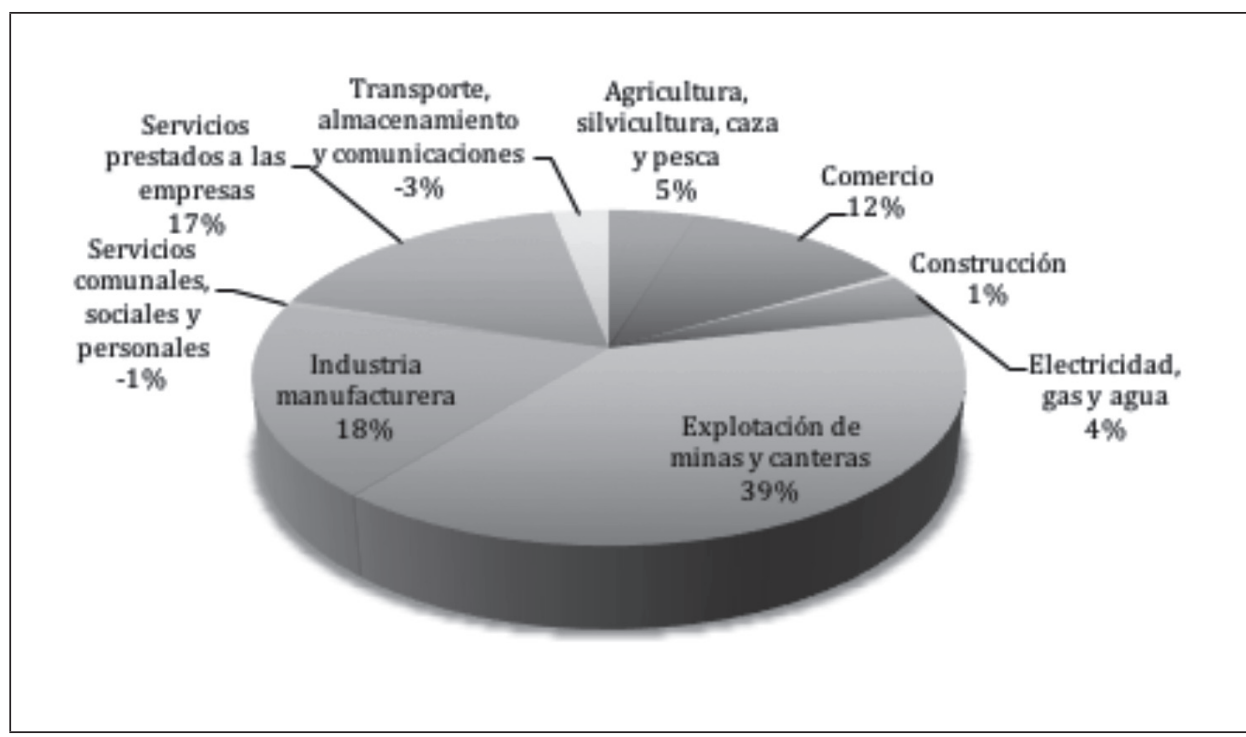

Fuente: Elaboración propia con base en Estadísticas Económicas del Ecuador-Sector Externo. Banco Central del Ecuador. Recuperado a partir de https://www.bce.fin.ec/index.php/component/k2/item/762

6 En el momento de escribir el presente artículo (marzo 2017) no habían sido publicados por el BCE datos del cuarto trimestre de 2016. 
Igualmente, la rama minera ha sido la que ha acaparado de manera mayoritaria la inversión extranjera directa de los últimos años. Así, en 2015 se invirtieron en este sector 559,8 millones, muy lejos de otros campos como el comercio (172,8 millones de dólares), manufacturas (264,101 millones de dólares) o servicios prestados a las empresas (243,2 millones de dólares). Esa tendencia se mantuvo en los tres primeros trimestres de 2016, cuando la minería y las canteras aglutinaron un monto de IED de 160,2 millones de dólares, seguido del comercio (77 millones) y el transporte (33,8 millones) (Banco Central del Ecuador, 2017a).

Estados Unidos con 186 millones de dólares y República Popular China con 113,8 millones reúnen el 22,7\% de toda la IED que llega a Ecuador, siendo América Latina (38,7\%, 511,1 millones de dólares) y Europa (35,2\%, 464,6 millones de dólares) los principales inversores en el país andino. Las dos naciones muestran comportamientos dispares en lo que se refiere a la elección de las actividades económicas a las que dirigen sus capitales. Mientras que China prefiere el sector minero (92 millones de dólares, un 81,59\% del total), EE.UU. se inclina hacia los servicios prestados a las empresas (165,8 millones de dólares, lo que supone un $89,7 \%$ del total).

La fijación por un único sector, por parte de las empresas chinas, respondería a la búsqueda de la satisfacción de sus necesidades económicas. El gigante asiático ha experimentado un rápido incremento de su capacidad industrial en las últimas décadas, solo este sector representó en 2016 el 40,9 \% de su PIB según el Banco Mundial (2017a), y para ello ha requerido de buscar materias primas para alimentarlo. De acuerdo con datos del sistema wITs, China importó en 2015 1,68 billones de dólares, de los que 366.439 millones correspondieron a estos componentes primarios, siendo los principales socios comerciales en este rubro países de América Latina (26,45\%) y Caribe y África (12,85\%), destacándose Brasil, Chile, Perú y Sudáfrica entre los 10 principales proveedores de los recursos mencionados para la segunda economía del mundo (wITs, 2017).

La inversión china acumulada en Ecuador desde 2009 sumó en 201511.000 millones de dólares, y está presente no solo en el sector de la minería, sino en otros como energía hidroeléctrica y eólica, petróleo y obra civil (carreteras, puentes y trabajos hidráulicos). Algunos de los proyectos en los que hay presencia china son las hidroeléctricas de Coca Codo Sinclair, construida por Sinohydro por valor de 2.200 millones de dólares; Sopladora, construido por Gezhouba, y Toachi Pilatón, construida por The China International Water and Electric Corp con financiación rusa. Además, el proyecto minero de cobre de El Mirador, donde participa CRCC-Tongguan Investment tras abonar 100 millones de dólares por los derechos sobre la mina bajo el compromiso de invertir 1.400 millones de dólares en cinco años, y en petróleo, las empresas CNPC y Sinopec, así como el consorcio Andes Petroleum que gestionan en Sucumbíos varios proyectos. En cambio, en Orellana y Pastaza algunas concesiones están comandadas por Petro Oriental y Andes Petroleum.

Asimismo, se está en conversaciones para que capital chino financie uno de los proyectos 
emblema de la Administración de Rafael Correa, la refinería del Pacífico, que inicialmente iba a levantarse con fondos venezolanos, de hecho, PSVA tenía el 49\% del consorcio constructor en asociación con Petroecuador, y que no acaba de concretarse (Krauss \& Bradsher, 2015). Otras inversiones del gigante asiático se localizan en energía eólica ${ }^{7}$, carreteras ${ }^{8}$, así como el consorcio de las empresas chinas Gezhouba, Hydrochina y China CAmc Engineering que estuvo a cargo del reencauce de los ríos Bulubulu, Cañar y Naranjal con un costo de 55,6 millones de dólares. En total, hasta el 2015 se habían invertido por parte de República Popular China 11.000 millones de dólares en Ecuador (Krauss \& Bradsher, 2015).

En cuanto a Estados Unidos de América, la preferencia por el área servicios reside en la gran competitividad de sus empresas de sectores, en especial aquellas con un gran componente tecnológico. Solo los servicios de comunicaciones y computación $(42,12 \%$ de todas las exportaciones totales de EE.UU. en este rubro) y los relacionados con las tecnologías de la información y la comunicación $(22,71 \%)$ suponen más de la mitad de las exportaciones de servicios norteamericanos. Otros sectores de servicios dinámicos del país norteamericano son los de viaje $(27,24 \%)$, seguros y servicios financieros (16,37\%), así como de transporte
$(11,94 \%)$ de acuerdo con los datos del sistema wITs (wITs, 2017). Lamentablemente no se ha podido encontrar, al contrario que en el caso chino, información pública o privada relativa a qué empresas norteamericanas están presentes en Ecuador.

\subsection{Deuda}

En el período de estudio Ecuador ha implementado un ambicioso programa de desarrollo del país centrado en infraestructuras de generación de energía, transporte y comunicaciones, así como servicios de educación y sanidad (fundamentalmente unidades educativas del Milenio, proyectos de universidades públicas punteras como Yachay, hospitales y otros centros médicos estatales). Este programa de desarrollo ha llevado a Ecuador a salir al exterior para buscar financiación, aumentando de este modo la deuda del país que pasó de un 27,2\% al 39,6\% en 2016 según el Ministerio de Finanzas ("Deuda Pública - Ministerio de Finanzas", s. f.) aproximándose al límite que el Código Orgánico de Planificación y Finanzas Públicas de la República fijó en el $40 \%$ del PIB en su artículo 124 (Ministerio de Finanzas, 2010).

La deuda externa del Ecuador, a diciembre de 2016, ascendió a 38.136,6 millones

\footnotetext{
Xinjiang Goldwind construyó un parque eólico en Villonaco de 16.5 megavatios que ya está operando desde hace cuatro años.

8 La misma Sinohydro está reconstruyendo y modernizando varias vías en Azuay y Morona Santiago, mientras que el puente de dos kilómetros de largo y cuatro carriles sobre el río Babahoyo fue construido por Guangxi Road \& Bridge Engineering Corp.
} 
repartidos en los siguientes rubros: $8.247,8$ millones de dólares correspondieron a organismos internacionales de financiación como el Banco Mundial, el Banco Interamericano de Desarrollo (BID) y la Corporación Andina de Fomento (CAF); 7.997,9 millones de dólares fueron préstamos gubernamentales y 8.844,6 restantes pertenecieron a entidades bancarias y financieras como Goldaman Sach, Citigroup Global Markets o Noble Americas Corp, a lo que se debe añadir la deuda privada del país, que sumó 7.801.972 millones de dólares (Banco Central del Ecuador, 2017a).

\section{Gráfico 2 \\ La deuda externa de Ecuador en el 2016 por acreedor en el período enero-diciembre 2016 (en millones de dólares)}

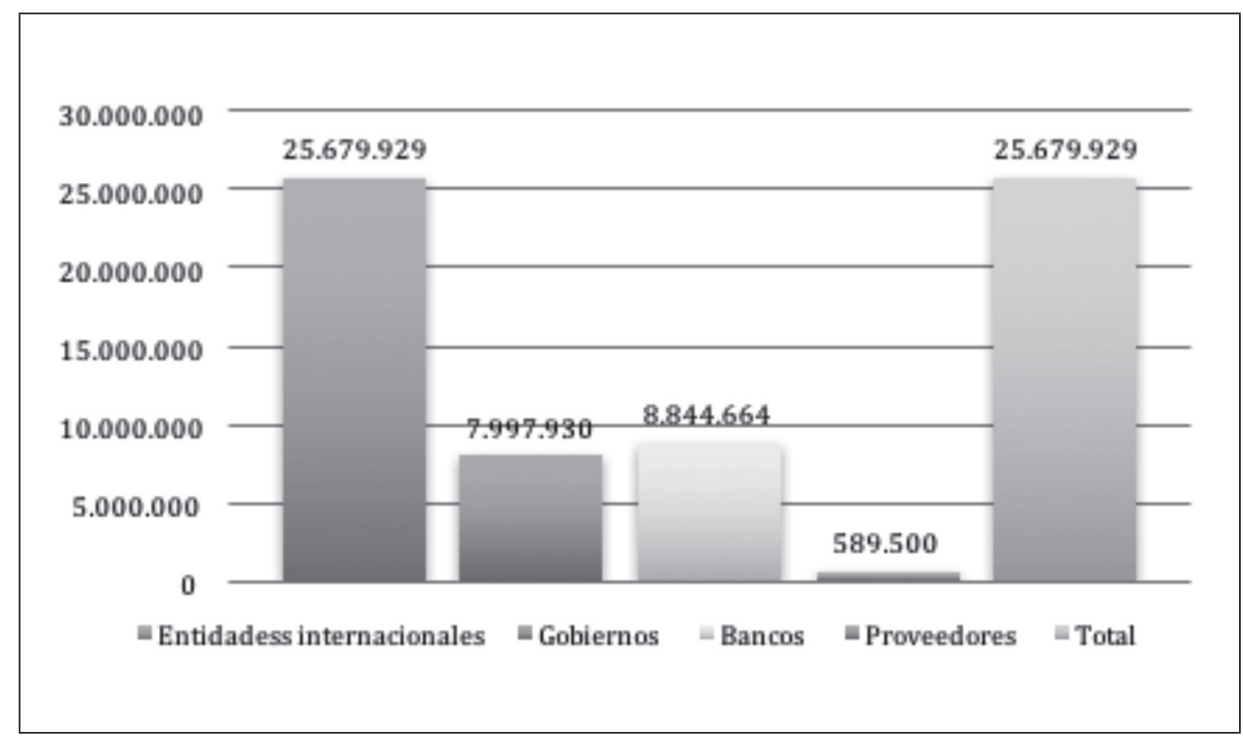

Fuente: Elaboración propia con base en Estadísticas Económicas del Ecuador-Sector Externo. Banco Central del Ecuador. Recuperado a partir de https://www.bce.fin.ec/index.php/component/k2/item/762

Por países, Ecuador tiene fuertes compromisos crediticios adquiridos fundamentalmente con China, que a enero de 2017 había prestado al país andino 8.272,8 millones de dólares. El equivalente al 31,3\% del total de su deuda. Muy lejos está como prestamista EE.UU., con solo $0,2 \%$ de la deuda total $(54,7$ millones de dólares), mientras que el segundo mayor socio financiero de Ecuador es España con 300,1 millones de dólares («Deuda Pública - Ministerio de Finanzas», s. f.). La deuda podría haber aumentado de concretarse las negociaciones, anunciadas por Rafael Correa en marzo de 2017, para la concesión de un nuevo présta- 
mo por parte del país asiático por importe de 1.000 millones de dólares, a 20 años de plazo y al $2 \%$ de interés, que iría destinado para la reconstrucción de las zonas afectadas por el terremoto que sufrió Ecuador en abril de 2016 (Agencias, 2017).

El endeudamiento con República Popular China no es un fenómeno único de Ecuador, pues el dinero de ese país supone una fuente crítica de liquidez para algunas naciones de la región latinoamericana, especialmente la de aquellas que en los últimos años han tenido un acceso limitado a mercados internacionales de capitales. De acuerdo con el informe reciente del centro de estudios The Dialogue, el 92\% de los créditos chinos se dirigieron hacia Venezuela, Ecuador, Brasil y Argentina (Myers \& Gallagher, 2017). Estos créditos, canalizados fundamentalmente a través de instituciones financieras públicas como el Chinese Development Bank y el China Eximbank, no poseen, al contrario que los procedentes de Estados occidentales, condicionalidades de tipo político, pero sí otras relativas a la contratación de empresas chinas o el empleo de equipamiento procedente de ese país (Myers \& Gallagher, 2017). Por otra parte, las tasas de interés de estos préstamos son relativamente elevados, entre el 6,87\% y el 7,25\%, y en el caso ecuatoriano están negociados de tal forma que el Estado andino devuelve el dinero a través de preventas petroleras (Araujo, 2016). Sin embargo, algunos de los contratos de preventas petroleras han venido siendo fuertemente cuestionados por el secretismo de los mismos; si bien ya se han dado a conocer las condiciones de algunos de ellos (Orozco, 2018), que parecieran apuntar a negociaciones poco favorables para Ecuador; en algún caso se está procediendo a la renegociación de los mismos con la contraparte china (El Comercio, 2018). Esto sin duda tiene un impacto en los márgenes que tanto el anterior ejecutivo como el actual gobierno de Lenin Moreno tengan, tanto en su planificación económica como en la política.

\subsection{Ayuda Oficial al Desarrollo (AOD)}

Ecuador desde el 2012 ha pasado a estar considerado por el Banco Mundial (BM) como país de renta media alta (upper-middle income), por lo que desde la fecha y hasta el presente los fondos recibidos en forma de Ayuda Oficial al Desarrollo (AOD) se han reducido. Según cifras manejadas por el BM, 2014 fue el último ejercicio en que Ecuador registró entradas de AOD neta recibida 9 por una suma de 160.380 .000 dólares, de los cuales 115 millones correspondieron a créditos no reembolsables y 70 millones a cooperación técnica (Banco Mundial, 2017c).

Por países, en 2014 los mayores contribuyentes de AOD fueron Estados Unidos de América (32,2 millones), la Unión Europea (22,8 millones), Alemania (36,4 millones) y Francia (40,1 millones). Así, solo entre la UE y los aportes individuales de sus Estados Ecuador recibió 99,3 millones de dólares (Banco Mundial, 2017d).

Europa ha sido en AOD un apoyo muy importante, y este tipo de flujos económicos

$9 \quad$ En dólares a precios actuales. 
ha contribuido, entre otros factores, a mejorar todos los indicadores de desarrollo socioeconómicos, como lo demuestra que el porcentaje de población pobre haya descendido de un $64,4 \%$ en 2000 a un $23,3 \%$ en 2015 (Banco Mundial, 2017b).

\section{CONCLUSIONES}

Gracias al análisis realizado, y basándonos en las tres variables económicas apuntadas por Hey, podemos establecer una serie de conclusiones muy relevantes.

En primer lugar, Estados Unidos de América sigue siendo el principal accionista comercial de Ecuador. Mediante el comercio exterior se observa una fuerte dependencia del país andino de su mercado, tanto para los exportadores (1.877 en 2016) como para los importadores (8.167 en 2016). No obstante, la relación puede considerarse, ahora, como equilibrada en tanto las cifras muestran una diferencia relativamente corta, seis puntos porcentuales, entre lo exportado y lo importado. Esto ha sido posible gracias, en parte, a una cierta diversificación del portafolio de mercados en los que sus productos están presentes. Asimismo, cabe destacar que el liderazgo norteamericano en el marco de la AOD prevaleció únicamente hasta el 2014.

Por otro lado, República Popular China es el mayor acreedor individual del país latinoamericano y uno de los valedores más im- portantes de su economía hoy en día. Además, China se revela como un actor importante para el sector importador ecuatoriano, compuesto por 6.423 empresas en 2016 según datos del Banco Central (Banco Central del Ecuador, 2017a). Los bajos precios de los artículos de origen y la facilidad para colocarlos entre los consumidores, precisamente por su accesibilidad, ha hecho que muchos comerciantes de múltiples sectores busquen proveedores orientales a costa de un abultado déficit comercial con esta nación, que representa un problema más para una ya de por sí complicada coyuntura económica para Ecuador.

En relación con la deuda puede bien afirmarse que Ecuador tiene una fuerte dependencia del dinero procedente de los bancos públicos controlados por el régimen de Beijing. Las causas de este acercamiento parecen residir en varios aspectos. El primero fue la decisión del presidente de la República de cerrarse y alejarse de las instituciones financieras de Bretton Woods hasta fechas recientes por considerar que sus políticas, seguidoras del denominado consenso de Washington, iban en contra de los intereses de la nación ${ }^{10}$. El segundo factor fue la cercanía ideológica con los líderes chinos con respecto al sistema y la gobernanza internacionales, así como el poco interés de estos por la presencia de cláusulas de tipo democrático o humanitario en los contratos de crédito. Por último, el tercero resultaría ser la necesidad de buscar liquidez desde el desplome

10 En 2015 Rafael Correa volvió a admitir la presencia de personal del FMI en Quito para supervisar la economía de la nación, uno de los requerimientos de esta entidad para prestar fondos, y actualmente el Fondo ha entregado créditos para paliar los efectos del terremoto del 2016. 
de los precios del petróleo de los últimos dos años y medio, que ha golpeado duramente a la economía ecuatoriana.

En segundo lugar, la comprobación del objetivo marcado llevó a verificar la hipótesis establecida, dado que la política exterior ecuatoriana en el marco temporal establecido resultó bicéfela dependiente; para cada una de ellas habría tenido en apariencia un comportamiento diferente, bien pudiendo encajar con lo que el corpus teórico identifica como de tipo dependiente (Hey 1993, 1994, 1995a, 1995b).

De esta manera, cabe plantearse que en política comercial existe una dependencia comercial de Ecuador con EE.uU. y China, al sumar entre ambos el $36,1 \%$ de las exportaciones globales y el 40,6\% de las importaciones totales. Son dos mercados importantes para el gobierno, aunque las relaciones en el ámbito político no han ido parejas a la relevancia de ambos. Mientras que con Washington ha primado una postura de distanciamiento y delimitación de barreras rojas muy claras en los términos de su relación comercial, defensa de la soberanía nacional y primacía del hombre en los asuntos económicos ${ }^{11}$ (ver Asamblea Constituyente, 2008, arts. 415 y sig.), con China ha existido un mayor acercamiento a alto nivel, incluidas visitas de los líderes de ambos países ${ }^{12}$ y firmas de convenios bilaterales, destacando el Convenio de Cooperación Económica y
Técnica rubricado en 2009 y ratificado por la Asamblea Nacional el 2013, que contempla entre otros asuntos la concesión de una línea de crédito china por valor de $20.000 .000 \mathrm{mi}-$ llones de yuanes para la financiación de proyectos acordados entre ambos gobiernos en el período 2009-2014 (Asamblea Nacional del Ecuador, 2013).

Visto lo anterior, se puede afirmar que bajo el mandato de Rafael Correa, Ecuador presentó dependencia económica no solo frente a EE.UU., sino también con China. Igualmente, la tradicional relación de Quito con su socio norteamericano, sea en el contexto comercial, inversor o de ayuda oficial de desarrollo, ha sido alterada por la presencia de un nuevo actor en la región, el gigante asiático.

En tercer lugar, y examinando las principales razones de cambio en el contexto mencionado, podemos destacar que el progresivo acercamiento entre Ecuador y Beijing es el resultado de una serie de variables de carácter ideológico, político y económico que identificaron el gobierno de Rafael Correa. Al contrario, el mantenimiento de los lazos económicos entre Quito y Washington se debe principalmente a la vinculación del sector empresarial entre ambos socios, así como al fenómeno migratorio ecuatoriano hacia el país norteño.

Por último, a través del estudio ejecutado pudimos profundizar en el análisis de la política

11 En el marco de la Cumbre de Naciones Unidas para la Aprobación de la Agenda para el Desarrollo después de 2015, celebrada en Nueva York en septiembre de 2015, el presidente propuso generar "una agenda de desarrollo donde el sentido de humanidad se imponga sobre el imperio del capital", resaltando ante el resto de países del sistema internacional su visión humanista y no economicista (Correa, 2015).

12 Rafael Correa viajó a China en enero de 2015, visita que fue devuelta por el máximo mandatario chino, Xi Jinping, en noviembre de 2016 (Telégrafo, 2016). 
exterior ecuatoriana desde el prisma de la teoría de política exterior dependiente (Dependent Foreign Policy Theory). De esta forma, y dentro del entorno descrito, observamos que el comportamiento de Ecuador en el período 2007-2017, en especial hacia su socio asiático, ha sido marcado por una dependencia económica y no fue tanto el resultado de una nueva política exterior subordinada a la relación con Estados Unidos.

En este sentido, cabría afirmar que el presidente de un país dependiente ve reducido su margen de maniobra, como se ha evidenciado para el caso de Rafael Correa al respecto de los contratos de construcción de grandes obras con capital e ingeniería china y de los préstamos bancarios de ese país, en ambos casos sujetos a condiciones no igualitarias para la república andina.

\section{REFERENCIAS}

Agencias. (23/03/2017). Ministro de Finanzas irá a China por nuevo préstamo. El Universo. Quito. Recuperado a partir de http://www.eluniverso.com/ noticias/2017/03/23/nota/6103159/finanzas-irachina-nuevo-prestamo

Andrade Zambrano, C. D. \& Ruperti León, L. (2016). Una mirada. República de Ecuador. Dominio de las Ciencias, vol. 2, No. 3, 55-66.

Araujo, A. (2016, abril de). La deuda con China sube a USD 8395 millones. El Comercio. Quito. Recuperado a partir de http://www.elcomercio. com/actualidad/deudachinaecuadorpetroleocredito.html

Asamblea Constituyente. Constitución del Ecuador. (2008). Recuperado a partir de http://www. asambleanacional.gob.ec/sites/default/files/pri- vate/asambleanacional/filesasambleanacionalnameuid-20/transparencia-2015/literal-a/a2/ Const-Enmienda-2015.pdf

Asamblea Nacional del Ecuador. (12/03/2013). Convenio de Cooperación Económica y Técnica entre la República del Ecuador y la República Popular China. Recuperado 16 de marzo de 2017, a partir de http://www.asambleanacional.gob.ec/es/ tratados-instrumento-internacionales-2013-2017

Banco Central del Ecuador. (2017a). Estadisticas Económicas del Ecuador-Sector Externo. Recuperado a partir de https://www.bce.fin.ec/index.php/ component $/ \mathrm{k} 2 /$ item $/ 762$

Banco Central del Ecuador. (2017b). Evolución de la Balanza Comercial. Balanza Comercial del Ecuador por Paises, enero-diciembre 2016. Quito: Banco Central del Ecuador. Recuperado a partir de https://contenido.bce.fin.ec/documentos/ Estadisticas/SectorExterno/BalanzaPagos/balanzaComercial/ebca201702.pdf

Banco Mundial. (2017a). Datos-China. Banco Mundial. Recuperado a partir de http://datos.bancomundial.org/pais/china?view=chart

Banco Mundial. (2017b). Datos-Ecuador. Banco Mundial. Recuperado a partir de http://datos.bancomundial.org/pais/ecuador?view=chart

Banco Mundial. (2017c). World Development Indicators: Aid dependency. Banco Mundial. Recuperado a partir de http://wdi.worldbank.org/table/6.11\#

Banco Mundial. (2017d). World Development Indicators: Distribution of net aid by Development Assistance Committee members. Banco Mundial. Recuperado a partir de http://wdi.worldbank.org/table/6.12\#

Bartolomé Puerto, V. A. (1991). Apertura exterior y dependencia económica: el caso de España. Espańa: Universidad de Barcelona.

Bonilla, A. \& Páez, A. (2006). Estados Unidos y la región andina. Nueva Sociedad, No. 206, 126-139. 
Borrego Pla, M. (1991). Dependencia económica e intervencionismo. Historia de las Américas, vol. 4, 113-130.

Cardoso, F. H. (1994). ¿"Teoría de la dependencia” o análisis concreto de situaciones de dependencia? Politica y Sociedad, No 1, 5-27.

Carrión Mena, F. (2007). Ecuador ¿planificación democrática y consensuada de su política exterior? Quórum: revista de pensamiento iberoamericano, No 17, 111-120.

Comercio, El (28/01/2018). Gobierno y petroleras asiáticas alcanzan acuerdos en renegociación de contratos de preventa. Recuperado 19 de febrero 2018 a partir de http://www.elcomercio.com/actualidad/negocios-gobierno-petroleras-asiaticasacuerdos.html

Correa, R. (27/09/2015). Intervención en Cumbre de NN.UU. para la aprobación de la Agenda para el Desarrollo después del 2015. Recuperado el 20 de enero de 2018 a partir de http://bit.ly/2CzqorG

Deuda Pública - Ministerio de Finanzas. (s.f.). Recuperado a partir de http://www.finanzas.gob.ec/ deuda-publica/

Erthal Abdenur, A. \& Marcondes de Souza Neto, D. (2013). Cooperación china en América Latina. Las implicaciones de la asistencia para el desarrollo. Íconos: Revista de Ciencias Sociales, No. 47, 69-85.

Fondo Monetario Internacional. (2017). IMF Data-China, P.R.: Mainland. Fondo Monetario Internacional. Recuperado a partir de http:// data.imf.org/?sk=85b51b5a-b74f-473a-be1649f1786949b3

Hey, J. A. K. (1993). Foreign Policy Options under Dependence: A Theoretical Evaluation with Evidence from Ecuador. Journal of Latin American Studies, 25(03), 543. https://doi.org/10.1017/ S0022216X00006660
Hey, J. A. K. (1994). Compliance, consensus and counter-dependence: Foreign policy in Ecuador. International Interactions, 19(3), 241-261. https://doi. org/10.1080/03050629408434829

Hey, J. A. K. (1995a). Foreign Policy in Dependent States. En L. Nedck, J. A. K. Hey \& P. J. Haney (Eds.), Foreign Policy Analysis: Continuity and Change in Its Second Generation (pp. 201-213). New Yersey: Prentice Hall.

Hey, J. A. K. (1995b). Theories of Dependent Foreign Policy and the case of Ecuador in the 1980s. EE.UU.: Ohio University Press. Recuperado a partir de http://gen.lib.rus.ec/book/index.php?md5=D 5425A31DCBE389A2484A2BA08A3087D

Hidalgo Capitán, A. L. (2012). Economía política del desarrollo y el subdesarrollo. Revisitando la Teoría de la Dependencia. Revista Iberoamericana de Estudios de Desarrollo, No 17, 107-116.

International Chamber of Commerce (Ed.). (2010). Incoterms ${ }^{\oplus}$ 2010: ICC rules for the use of domestic and international trade terms; entry into force: 1 january 2011. Paris: ICC Service Publications.

Kay, C. (1988). Estructuralismo y teoría de la dependencia en el período neoliberal. Una perspectiva latinoamericana. Nueva Sociedad, No. 158, 100-119.

Krauss, C. \& Bradsher, K. (22/07/2015). Con préstamos y exigencias, China expande su influencia en América Latina. The New York Times. El Chaco-Ecuador. Recuperado a partir de https:// www.nytimes.com/2015/07/26/universal/es/ con-prestamos-y-exigencias-china-expande-suinfluencia-en-ecuador-y-el-resto-de-americalatina.html?mwrsm=Email\&_r=1

Lauderbaugh, G. M. (2009). Estados Unidos y Ecuador durante la segunda guerra mundial: conflicto y convergencia. En Ecuador: relaciones internacionales a la luz del bicentenario (pp. 265-296). Quito: Flacso Ecuador. 
Malamud Rikles, C. D. \& García-Calvo Rosell, C. (2009). Análisis del Real Instituto Elcano, No. 61.

Mazzoni, M., Schleifer, P. \& García A. (2013). Capitalismo y Estado reflexiones desde la teoría de la dependencia en América Latina. Kairos: Revista de Temas Sociales, No. 31, 1-15.

Ministerio Coordinador de Sectores Estratégicos. (2016). Agenda Nacional de la Energía 2016-2040. Quito: Ministerio Coordinador de Sectores Estratégicos. Recuperado a partir de http://www. sectoresestrategicos.gob.ec/wp-content/uploads/ downloads/2016/10/AGENDA-DE-ENERGIA2016-2040-vf.pdf

Ministerio de Finanzas. Código Orgánico de Planificación y Finanzas Públicas. (2010). Recuperado a partir de http://www.finanzas.gob.ec/wp-content/uploads/downloads/2012/09/CODIGO_ PLANIFICACION_FINAZAS.pdf

Ministerio de Relaciones Exteriores, Comercio e Integración de la República de Ecuador. (2007). Plan Nacional de Desarrollo 2007-2010. Politica Exterior. Quito.

Ministerio de Relaciones Exteriores de la República de Ecuador. (2006). Planex 2020. Plan Nacional de Politica Exterior 2006-2020. Quito.

Myers, M. \& Gallagher, K. (23/02/2017). Chinese Finance to LAC in 2016. Recuperado a partir de http://www.thedialogue.org/wp-content/ uploads/2017/02/Chinese-Finance-to-LAC-in2016-Web-and-email-res.pdf

Nacht, P. A. (2013). El Dragón en América Latina. Las relaciones económico-comerciales y los riesgos para la región. Íconos: Revista de Ciencias Sociales, No. 45, 141-154.

Orozco, M. (14/02/2018). Ecuador vendió incluso el petróleo que no tenía en 2016. El Comercio. Recuperado 19 de febrero de 2018, a partir de http://www.elcomercio.com/actualidad/ecuadorventa-petroleo-petrotailandia-petroecuador.html.

Ovando Santana, C. \& Aranda Bustamante, G. (2013). La autonomía en la política exterior latinoamericana. Evolución y debates actuales. Papel Politico, vol. 18, No. 2, 719-742.

Patińo, R. (2010). Discurso de posicionamiento del economista Ricardo Patińo como ministro de relaciones exteriores, comercio e integración. Recuperado el 19 de febrero de 2018 a partir de http://www. cancilleria.gob.ec/discurso-de-posicionamientodel-economista-ricardo-patino-como-ministrode-relaciones-exteriores-comercio-e-integracion/

Penalosa, R. J. (1980). El concepto de dependencia económica y su futuro. ICE: Revista de Economía, No 564-565, 137-146.

Pineo, R. (2009). Las relaciones entre Ecuador y Estados Unidos durante la guerra fría, del fin de la década del cuarenta a inicios de los ańos sesenta. En Ecuador: relaciones internacionales a la luz del bicentenario (pp. 297-330). Quito: Flacso Ecuador.

Ray, R.; Gallagher, K. \& Sarmiento, R. (2016). Boletin Económico China-América Latina 2016 (Research from the global economic governance initiative, No. 2016-3) (p. 13). Boston University -Research from the global economic governance initiative. Recuperado a partir de https://www.bu.edu/ pardeeschool/files/2016/04/Economic-Bulletin2016-Spanish.Final_.pdf

Samanamud, G. T. (2014). China en América Latina: los casos de Ecuador y Perú entre los años 20092012, ¿es posible una apuesta hacia el futuro? Anuario Mexicano de Derecho Internacional, No. 14, 221-260.

Sánchez, F. (2017). Un balance no convencional de Correa en Ecuador. Politica Exterior, vol. 31, No $176,146-154$ 
Sánchez Padilla, A. (2016). ¿ En defensa de la Doctrina Monroe? Los desencuentros en América Latina entre España y Estados Unidos (1880-1890). Historia Critica, No. 40, 62, 13-33. https://doi. org/10.7440/histcrit62.2016.01

Secretaría Nacional de Planificación y Desarrollo (Ed.). (2013). Plan Nacional para el Buen Vivir 20132017. Quito. Recuperado a partir de http://www. buenvivir.gob.ec/versiones-plan-nacional;jsession id=90B23E7A1A9EE3282A66F812AACB6105
Steans, J. (Ed.). (2010). An introduction to international relations theory: perspectives and themes (3. ed). Harlow: Pearson Education.

Telégrafo, El. (17/11/2016). Sin duda, China ha ayudado a transformar la historia del Ecuador. Recuperado 16 de marzo de 2017, a partir de http://www. eltelegrafo.com.ec/noticias/politica/2/presidentede-china-inicia-visita-de-estado-a-ecuador

Wits. (2017). China Datos básicos del comercio mundial: Valores más recientes. World Integrated Trade Solution. Recuperado a partir de http://wits.worldbank.org/countrysnapshot/es/CHN 